\title{
Ruptura bilateral simultânea do tendão quadricipital em paciente portador de diabetes mellitus do tipo II após trauma de baixa energia: Relato de caso*
}

\section{Quadricipital Tendon Simultaneous Bilateral Tear in a Patient with Type-II Diabetes Mellitus After Low-Energy Trauma: Case Report}

\author{
Breno Almeida de Pinho Tavares ${ }^{1}$ Leonardo Cortes Antunes ${ }^{1}$ Sara Johanna Sanchez Guerrero ${ }^{2}$

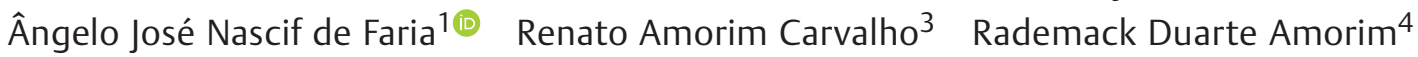

${ }^{1}$ Hospital São Francisco de Assis, Belo Horizonte, MG, Brasil

2 Departamento de Ortopedia e Traumatologia, Hospital São Francisco de Assis, Belo Horizonte, MG, Brasil

${ }^{3}$ Hospital Santa Lúcia Norte, Brasília, DF, Brasil

4 PRONTOMED, Teresina, PI, Brasil
Endereço para correspondência Ângelo José Nascif de Faria, Rua Ouro Preto 581/507, Belo Horizonte, MG, 30180-070, Brasil (e-mail: angelojnf@gmail.com).

Rev Bras Ortop

\begin{abstract}
Resumo
Palavras-chave

- joelho

- músculo quadríceps

- traumatismos dos tendões

- ruptura

A ruptura bilateral simultânea do tendão do quadríceps é uma lesão extremamente rara. Relatamos um caso desta lesão após trauma de baixa energia em paciente portador de diabetes mellitus do tipo II. O diagnóstico é essencialmente clínico, e requer tratamento precoce. Os dois joelhos foram abordados cirurgicamente no mesmo ato operatório. A reabilitação precoce é fundamental para a recuperação funcional adequada do joelho. O objetivo deste relato foi descrever um caso atípico deste tipo de lesão após trauma mínimo, bem como detalhar a técnica cirúrgica utilizada para o tratamento.
\end{abstract}
Abstract
Keywords
- knee
- quadriceps muscle
- tendon injuries
- rupture

Simultaneous bilateral rupture of the quadricipital tendon is an extremely rare lesion. We report a case of this injury after low-energy trauma in a patient with type-II diabetes mellitus. Both knees were surgically approached in the same surgical procedure. Early rehabilitation is essential for the adequate functional recovery of the knee. The aim of the present report was to describe an atypical case of this type of injury after minimal trauma, as well as to detail the surgical technique used to treat it.

Trabalho desenvolvido no Hospital São Francisco de Assis, Belo Horizonte, MG, Brasil.

recebido

14 de Setembro de 2020

aceito

14 de Outubro de 2020
DOI https://doi.org/

$10.1055 / \mathrm{s}-0040-1722584$. ISSN 0102-3616. (c) 2021. Sociedade Brasileira de Ortopedia e Traumatologia. All rights reserved.

This is an open access article published by Thieme under the terms of the Creative Commons Attribution-NonDerivative-NonCommercial-License, permitting copying and reproduction so long as the original work is given appropriate credit. Contents may not be used for commercial purposes, or adapted, remixed, transformed or built upon. (https://creativecommons.org/ licenses/by-nc-nd/4.0/)

Thieme Revinter Publicações Ltda., Rua do Matoso 170, Rio de Janeiro, RJ, CEP 20270-135, Brazil 


\section{Introdução}

A ruptura total do tendão do quadríceps é uma entidade bastante conhecida na ortopedia, e necessita de tratamento cirúrgico para o restabelecimento da função do mecanismo extensor. ${ }^{1}$ Esta lesão é uma condição rara. ${ }^{2}$ Descrita pela primeira vez por Steiner e Palmer ${ }^{3}$ em 1949, é mais comumente encontrada em pacientes maiores de 50 anos. Foram descritos casos desta lesão relacionados a patologias prévias, como artrite reumatoide, lúpus eritematoso sistêmico, arteriosclerose, diabetes mellitus (DM), hiperparatireoidismo primário e secundário, gota, tuberculose e vasculites, ${ }^{4}$ sendo a insuficiência renal crônica (IRC) o fator de risco mais encontrado nessas rupturas. ${ }^{5}$

\section{Descrição de Caso}

Paciente do sexo masculino, 66 anos, que apresentava queixa de dor aguda nos joelhos e inpacidade funcional. Ele relatou que, ao descer uma escada, realizou um salto ao solo, caiu com os pés apoiados e os joelhos fletidos, sentiu subitamente dor bilateral no nível do joelho, e não conseguiu deambular. Ao exame físico, observou-se derrame articular $(++/ 4+)$ bilateral, presença de hiato na região do polo superior da patela no joelho direito, e na transição miotendinosa quadriciptal no esquerdo. Solicitou-se que realizasse, separadamente, elevação ativa dos membros inferiores em extensão, mas ele não conseguiu manter a posição contra a gravidade. Radiografias evidenciaram sindesmófito suprapatelar bilateral e rótula inferiorizada. No histórico do paciente, havia um relato de DM controlada com o uso de antiglicêmicos orais. Seu peso encontrava-se dentro da normalidade segundo o índice de massa corporal (IMC), e os exames laboratoriais apresentavam-se normais.

O paciente foi submetido a tratamento cirúrgico dois dias após a lesão. Os dois joelhos foram abordados no mesmo ato operatório. Sob raquianestesia e com o uso de dois torniquetes (inflados separadamente) na raiz das coxas, foi realizada incisão mediana anterior de cerca de $7 \mathrm{~cm}$ de distal para proximal a partir do polo superior da patela no joelho direito (-Figura 1 ). Após a exploracão cirúrgica, foi identificada desinserção completa do tendão do quadríceps na região proximal da rótula.

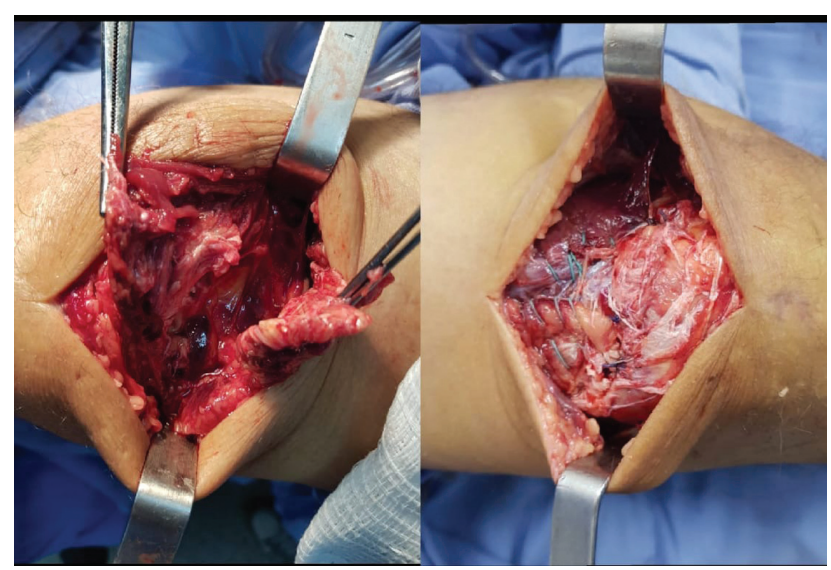

Fig. 1 Lesão do tendão quadriciptal do joelho direito e imagem radiográfica do pós-operatório.

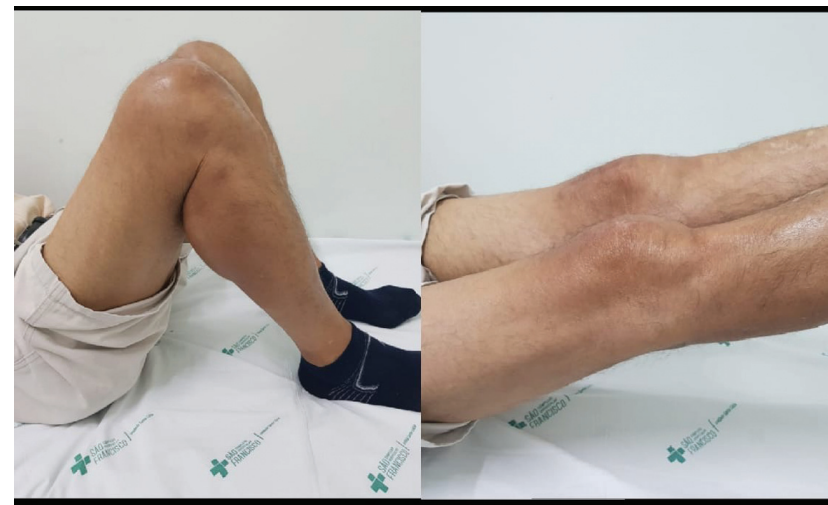

Fig. 2 Lesão do tendão quadriciptal do joelho esquerdo e sutura término-terminal do tendão do quadríceps.

Após a retirada do hematoma, foi realizado reparo do coto proximal ao polo superior da rótula com a técnica de Krackow, ${ }^{6}$ utilizando fios inabsorvíveis número 2 (Ethibond, Medline Industries, Inc., Northfield, IL, EUA). Também foram utilizadas 2 âncoras de $5 \mathrm{~mm}$ no polo superior da patela, realizando-se dessa forma a reinserção tendinosa, com o joelho em $40^{\circ}$ de flexão. A lesão do joelho esquerdo foi abordada com incisão semelhante, sendo observada uma lesão completa na junção miotendinosa do tendão do quadríceps e realizada a sutura término-terminal pela técnica de Krackow, ${ }^{6}$ utilizando fios Ethibond número 2, com o joelho em $40^{\circ}$ de flexão (-Figura 2 ). Em ambos, os joelhos as lesões retinaculares foram reparadas com Vycril 2.0 (Ethicon, Somerville, NJ, EUA).

No pós-operatório imediato, os joelhos foram imobilizados com talas gessadas por três semanas. Sete dias após a cirurgia, foram iniciados os exercícios isométricos do quadríceps. A reabilitação com fisioterapia autopassiva evoluiu com amplitude de movimento satisfatória. A carga com auxílio de andador foi permitida com seis semanas de evolução.

\section{Discussão}

Segundo Kellersmann et al., ${ }^{2}$ esta é uma entidade rara, geralmente relacionada à presença de doenças sistêmicas crônicas, ou ao uso de esteroides. O fator de risco mais encontrado é a IRC, conforme relataram Tomazini et al. ${ }^{7}$ De acordo com Kara et al,.. ${ }^{4}$ o mecanismo de trauma mais comum é a contração súbita e excêntrica do músculo quadríceps com flexão do joelho concomitante aos pés fixos ao solo.

Segundo Matokovic et al., 5 a DM é responsável por alterações funcionais e estruturais tanto na macrocirculação quanto na microcirculação tendinosa, acarretando anormalidades bioquímicas e estruturais em vários orgãos e tecidos, inclusive nos tendões. 0 trabalho de Sharma e Maffulli ${ }^{8}$ mostrou que as lesões endoteliais promovidas pela DM causam uma redução na síntese e secreção dos fatores protetores, levando às alterações que propiciam um estado pró-constritor, pró-inflamatório e pró-agregante ao vaso sanguíneo, gerando uma diminuição de aporte sanguíneo. Acreditamos que esta seja a principal explicação para a ruptura tendinosa descrita. 


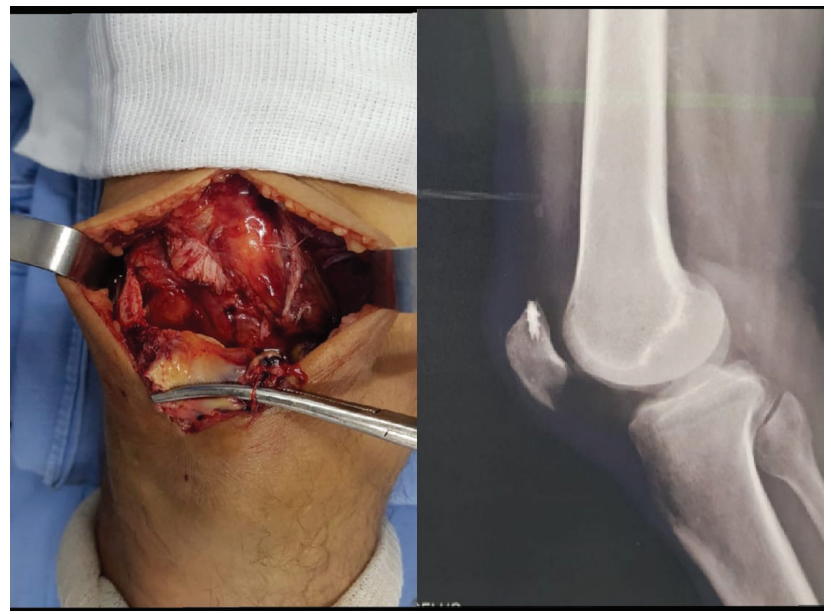

Fig. 3 Paciente com três meses de pós-operatório apresentando boa amplitude de movimento e função preservada do mecanismo extensor bilateralmente.

Para Ilan et al., ${ }^{9}$ o diagnóstico desta lesão é baseado em achados clínicos. Ao exame físico, o paciente geralmente apresenta um hiato palpável acima da patela, e a extensão ativa dos joelhos fica comprometida. Outros sinais incluem a hemartrose, a ausência de reflexos patelares, ou a presença de patela flutuante. Esses sinais e sintomas sugerem o diagnóstico da lesão. De acordo com Kim et al., ${ }^{10}$ as radiografias dos joelhos devem ser solicitadas; no entanto, podem mostrar apenas alguns sinais indiretos de ruptura. Em casos de dúvida diagnóstica ou lesões associadas, a ressonância magnética e a tomografia computadorizada podem ser úteis. As rupturas completas requerem tratamento cirúrgico, sendo a intervenção cirúrgica precoce um fator determinante para um bom resultado. O reparo cirúrgico tardio tem sido associado a piores resultados funcionais. A maioria dos pacientes com reparos bilaterais do tendão do quadríceps simultâneos podem esperar um bom resultado funcional; entretanto, muitos apresentarão dificuldade para retornar às atividades esportivas de alto nível.

A literatura ortopédica é escassa quanto aos relatos de ruptura bilateral simultânea do tendão quadricipital em paciente portador de DM. Os autores do presente estudo relataram um caso raro em que o paciente sofreu esta lesão após um trauma mínimo, cuja doença de base era a DM do tipo II.
Acredita-se que essa doença crônica seja uma causa importante de enfraquecimento da estrutura tendinosa quadriciptal, cujo principal motivo de ruptura seria a redução do aporte sanguíneo local devido às alterações na sua micro e macrocirculação. A natureza bilateral da lesão pode dificultar a reabilitação. As principais razões para os excelentes resultados funcionais incluem: reparo precoce do tendão com a tensão e força adequadas da sutura, o tempo reduzido de imobilização, e um programa de fisioterapia eficaz (-Figura 3).

\section{Conflito de Interesses}

Os autores declaram não haver conflito de interesses.

\section{Referencias}

1 Senevirathna S, Radha S, Rajeev A. Bilateral simultaneous rupture of the quadriceps tendon in a patient with psoriasis: a case report and review of the literature. J Med Case Reports 2011;5:331

2 Kellersmann R, Blattert TR, Weckbach A. Bilateral patellar tendon rupture without predisposing systemic disease or steroid use: a case report and review of the literature. Arch Orthop Trauma Surg 2005;125(02):127-133

3 Steiner CA, Palmer LH. Simultaneous bilateral rupture of the quadriceps tendon. Am J Surg 1949;78(05):752-755

4 Kara A, Sari S, Şeker A, Öztürk I. Osteotendinous repair of bilateral spontaneous quadriceps tendon ruptures with the Krackow technique in two patients with chronic renal failure. Acta Orthop Traumatol Turc 2013;47(01):68-71

5 Matokovic D, Matijasevic B, Petrić P, Crnkovic T, Skorvaga S. A case report of spontaneous concurrent bilateral rupture of the quadriceps tendons in a patient with chronic renal failure. Ther Apher Dial 2010;14(01):104-107

6 Krackow KA, Thomas SC, Jones LC. Ligament-tendon fixation: analysis of a new stitch and comparison with standard techniques. Orthopedics 1988;11(06):909-917

7 Tomazini GC, Carstens LD, Stieven Filho E, Castro Júnior MAT. Ruptura bilateral do quadríceps na doença renal Crônica. Rev Med UFPR1(04):162-165

8 Sharma P, Maffulli N. Tendon injury and tendinopathy: healing and repair. J Bone Joint Surg Am 2005;87(01):187-202

9 Ilan DI, Tejwani N, Keschner M, Leibman M. Quadriceps tendon rupture. J Am Acad Orthop Surg 2003;11(03):192-200

10 Kim YH, Shafi M, Lee YS, Kim JY, Kim WY, Han CW. Spontaneous and simultaneous rupture of both quadriceps tendons in a patient with chronic renal failure. A case studied by MRI both preoperatively and postoperatively. Knee Surg Sports Traumatol Arthrosc 2006;14(01):55-59 